\title{
Charge-Density-Wave-Induced Bands Renormalization and Energy Gaps in a Kagome Superconductor $\mathrm{RbV}_{3} \mathrm{Sb}_{5}$
}

\author{
Zhonghao Liu $\odot,{ }^{1,2, *}$ Ningning Zhao $\odot,{ }^{3}$ Qiangwei Yin, ${ }^{3}$ Chunsheng Gong, ${ }^{3}$ Zhijun $\mathrm{Tu}^{3}{ }^{3} \mathrm{Man} \mathrm{Li},{ }^{3}$ Wenhua Song, ${ }^{3}$ \\ Zhengtai Liu $\odot,{ }^{1}$ Dawei Shen, ${ }^{1,2}$ Yaobo Huang $\odot,{ }^{4}$ Kai Liu $\odot,{ }^{3, \dagger}$ Hechang Lei, ${ }^{3, \$}$ and Shancai Wang ${ }^{3, \$}$ \\ ${ }^{1}$ State Key Laboratory of Functional Materials for Informatics, Shanghai Institute of Microsystem \\ and Information Technology, Chinese Academy of Sciences, Shanghai 200050, China \\ ${ }^{2}$ College of Materials Science and Opto-Electronic Technology, \\ University of Chinese Academy of Sciences, Beijing 100049, China \\ ${ }^{3}$ Department of Physics and Beijing Key Laboratory of Opto-Electronic Functional \\ Materials \& Micro-Nano Devices, Renmin University of China, Beijing 100872, China \\ ${ }^{4}$ Shanghai Advanced Research Institute, Chinese Academy of Sciences, Shanghai 201204, China
}

(Received 20 April 2021; revised 19 July 2021; accepted 26 August 2021; published 14 October 2021; corrected 23 December 2021)

\begin{abstract}
Recently discovered $Z_{2}$ topological kagome metals $A \mathrm{~V}_{3} \mathrm{Sb}_{5}(A=\mathrm{K}, \mathrm{Rb}$, and Cs) exhibit charge-densitywave $(\mathrm{CDW})$ phases and novel superconducting paring states providing a versatile platform for studying the interplay between electron correlation and quantum orders. Here we directly visualize CDW-induced bands renormalization and energy gaps in $\mathrm{RbV}_{3} \mathrm{Sb}_{5}$ using angle-resolved photoemission spectroscopy pointing to the key role of tuning van Hove singularities to the Fermi energy in mechanisms of ordering phases. Near the CDW transition temperature, the bands around the Brillouin zone (BZ) boundary are shifted to high-binding energy, forming an M-shaped band with singularities near the Fermi energy. The Fermi surfaces are partially gapped, and the electronic states on the residual ones should be possibly dedicated to the superconductivity. Our findings are significant in understanding CDW formation and its associated superconductivity.
\end{abstract}

DOI: 10.1103/PhysRevX.11.041010

Subject Areas: Condensed Matter Physics Superconductivity

\section{INTRODUCTION}

Layered kagome-lattice transition metals are emerging as an exciting platform to explore frustrated lattice geometry and quantum topology. A set of typical kagome-lattice electronic bands is produced by the tight-binding calculation featuring a Dirac dispersion at the Brillouin zone (BZ) corner, a saddle point at the zone boundary, and a flat band through the BZ [1]. Close-totextbook kagome electronic bands with orbital differentiation physics have been experimentally observed in paramagnet CoSn [1]. In some kagome-lattice materials, the versatile quantum phenomena associating with the features near the Fermi energy $\left(E_{F}\right)$ have been found, such as Dirac and Weyl fermions [2-12], ferromagnetism

\footnotetext{
*1zh17@mail.sim.ac.cn

${ }^{\dagger}$ kliu@ ruc.edu.cn

thlei@ruc.edu.cn

§scw@ruc.edu.cn
}

Published by the American Physical Society under the terms of the Creative Commons Attribution 4.0 International license. Further distribution of this work must maintain attribution to the author(s) and the published article's title, journal citation, and DOI.
[13-15], negative flat-band magnetism [16], and the topological Chern magnet [17].

The theory was put forward early that a two-dimensional (2D) energy band with saddle points in the vicinity of $E_{F}$ is unstable against charge-density-wave (CDW) formation [18]. The CDW, superconducting, and topological phases have been extensively investigated in 2D transition-metal dichalcogenides [19], and the underlying microscopic mechanism of the CDW formation is still controversial. Recently, the CDW state and superconductivity were discovered in a family of layered kagome metals $A \mathrm{~V}_{3} \mathrm{Sb}_{5}(A=\mathrm{K}, \mathrm{Rb}$, and $\mathrm{Cs})$ [20-24], which hosts a $Z_{2}$ topological invariant and nontrivial topological Dirac surface states near $E_{F}$ [21]. The CDW state is probably driven by the competing electronic orders at the saddle-point singularity with a high density of states [25-39]. X-ray diffraction and scanning tunneling microscopy (STM) reveal the formation of a three-dimensional (3D) $2 \times 2 \times 2$ superlattice at both CDW and superconducting states [24-28], which energetically favors a chiral charge order and an inverse Star of David distortion in a kagome lattice with the shift of van Hove singularity to $E_{F}[34,35,40]$. The CDW states and double superconducting domes are associated with multiple singularities with different energies and orbital characters near $E_{F}$ [27-37,41-44], which at 

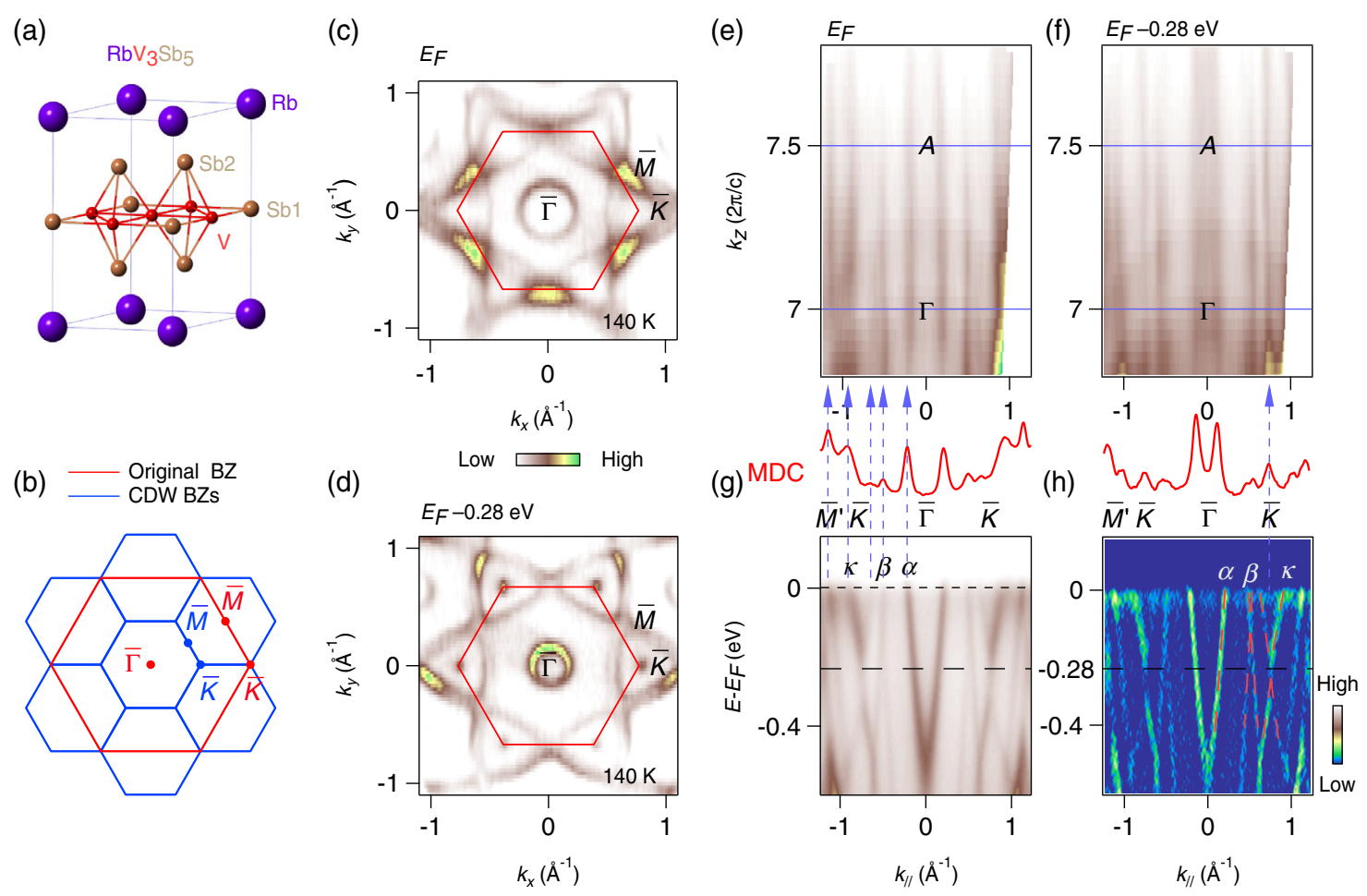

FIG. 1. (a) Crystal structure of $\mathrm{RbV}_{3} \mathrm{Sb}_{5}$ with space group $P 6 / \mathrm{mmm}$ (no. 191). (b) The original (red lines) and $2 \times 2$ reconstructed (blue lines) BZs projected on the (001) surface with the high-symmetry points. (c),(d) Integrated intensity plot ( \pm 10 meV) at $E_{F}$ and $E_{F}-0.28 \mathrm{eV}$ taken at $140 \mathrm{~K}$. The red lines indicate the high-symmetry directions and the original BZs. (e),(f) Integrated intensity plots $( \pm 10 \mathrm{meV})$ on the $k_{z}-k_{\|}$plane at $E_{F}$ and $E_{F}-0.28 \mathrm{eV}$ with $k_{\|}$oriented along the $\bar{\Gamma}-\bar{K}$ direction. The high-symmetry points are plotted. (g),(h) Intensity plot and corresponding second derivative plot along the $\bar{\Gamma}-\bar{K}$ direction. The MDCs taken at $E_{F}$ and $E_{F}-$ $0.28 \mathrm{eV}$ are shown by the red curves. The bands are indicated by the greek letters and the red dashed lines.

present need to be further studied in detail. In addition, a giant anomalous Hall effect with the reversal of the Hall sign is observed [22], and magnetic order and the local moment are not found by magnetic susceptibility and muon spin spectroscopy $[22,23,45]$. To fundamentally understand these anomalous behaviors and quantum orders, the investigation of the temperature evolution of the low-energy electronic structure is highly desired.

In this paper, we report on a combined angle-resolved photoemission spectroscopy (ARPES) and first-principles calculations study of the temperature evolution of the lowenergy electronic structure in $\mathrm{RbV}_{3} \mathrm{Sb}_{5}$, which has a CDW transition temperature $\left(T_{\mathrm{CDW}}\right)$ of about $102 \mathrm{~K}$, a sign change of the Hall coefficient at about $40 \mathrm{~K}$, and a superconducting transition temperature $\left(T_{\mathrm{c}}\right)$ of about $0.92 \mathrm{~K}[23]$. As a result of the CDW transition, we find that the bands at the zone boundary $(\bar{M})$ are shifted down about $40 \mathrm{meV}$ forming an $\mathrm{M}$-shaped band with its singularity at about $60 \mathrm{meV}$ below $E_{F}$. Below $T_{\mathrm{CDW}}$, the energy gap of about $20 \mathrm{meV}$ is opened at the Fermi momentum $\left(k_{F}\right)$ of the band centered at $\bar{M}$, and no gap is observed at the band centered at $\bar{\Gamma}$ at $10 \mathrm{~K}$ within experimental energy resolution. The electronic states on the residual Fermi surfaces should be dedicated to the superconducting pairing. Our findings reveal CDW-induced strong bands renormalization and energy gaps at the zone boundary, implying that they are the multiple singularities at $M$ which play ultimate roles in the formation of both CDW and its related superconducting phases.

\section{RESULTS AND DISCUSSION}

The crystal structure of $\mathrm{RbV}_{3} \mathrm{Sb}_{5}$ crystallizes in a hexagonal structure with $P 6 / \mathrm{mmm}$ (no. 191) space group [20-24], in which V-Sb slabs consisting of V kagome nets and interspersing $\mathrm{Sb}$ atoms are separated by alkali-metal ions along the $c$ axis, as shown in Fig. 1(a). There are two kinds of Sb sites: the $\mathrm{Sb} 1$ site at the centers of $\mathrm{V}$ hexagons, and the $\mathrm{Sb} 2$ site below and above the centers of $\mathrm{V}$ triangles forming hexagon layers. The corresponding original (red lines) and $2 \times 2$ reconstructed (blue lines) BZs projected on the (001) surface with the high-symmetry points are shown in Fig. 1(b). Figures 1(c) and 1(d) show constant-energy surfaces taken at $140 \mathrm{~K}$ at $E_{F}$ and $E_{F}-0.28 \mathrm{eV}$, respectively. The high intensity around the $\bar{M}$ points at the Fermi surfaces suggests the singularities or the surface states at the proximity of $E_{F}$. To investigate the 3D character of the Fermi surfaces, we carry out the photon-energy-dependent 

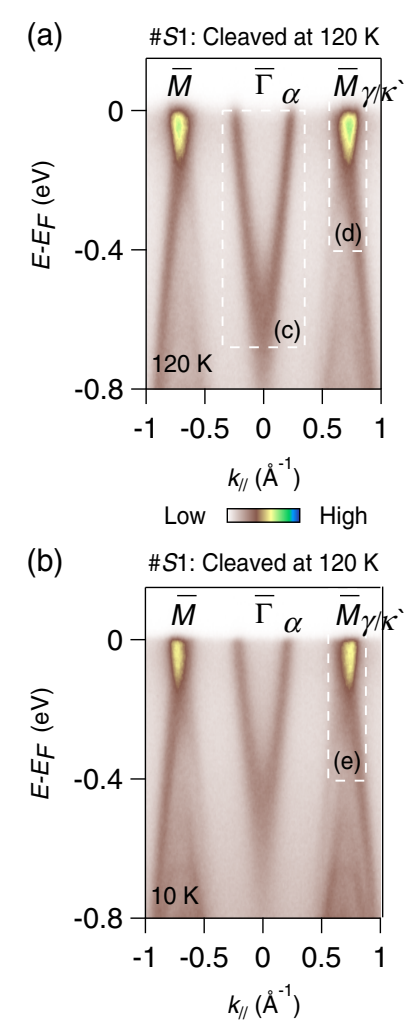

(c)

$(\mathrm{d})$

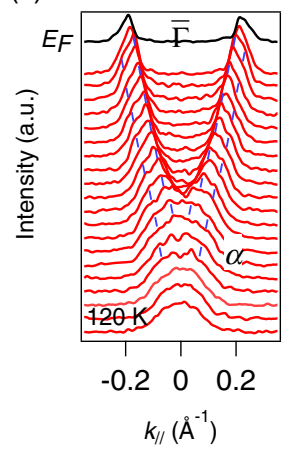

(f) $\quad$ EDCs at $\bar{M}$

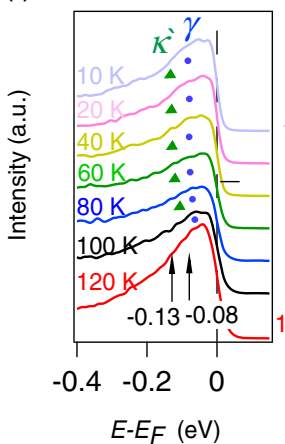

(d)

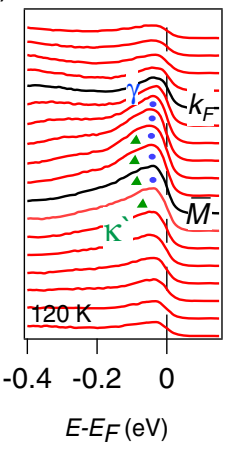

(e)

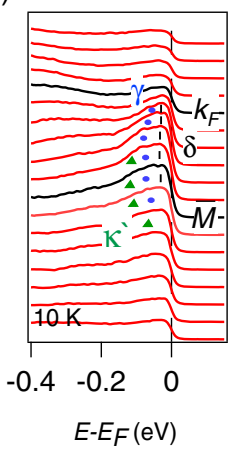

(g) Gaps at $k_{F} \quad(\mathrm{~h})$

(h) Gaps at $k_{F}$

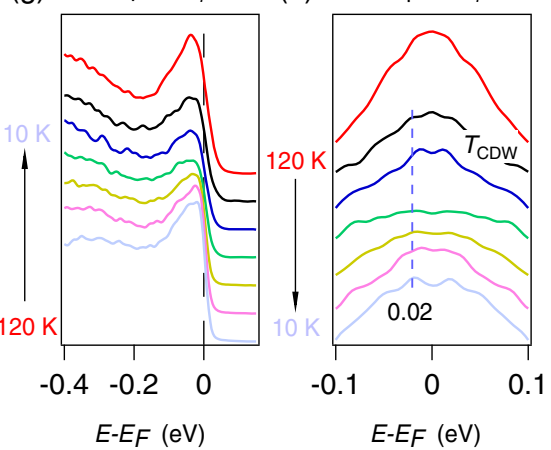

FIG. 2. (a),(b) Intensity plots along the $\bar{\Gamma}-\bar{M}$ direction taken at 120 and $10 \mathrm{~K}$. The sample (\#S1) is cleaved at $120 \mathrm{~K}$ and measured along with the decreasing temperature. The bands are indicated by the greek letters. (c) MDCs around the $\bar{\Gamma}$ point taken at $120 \mathrm{~K}$, as indicated by the dashed rectangles in (a). (d), (e) Energy distribution curves (EDCs) around the $\bar{M}$ point at 120 and $10 \mathrm{~K}$, as indicated by the dashed rectangles in (a) and (b). The $\kappa^{\prime}$ and $\gamma$ bands are indicated by different color makers. (f) EDCs at the $\bar{M}$ center taken at different temperatures. (g),(h) EDCs and their symmetrizations at the $k_{F}$ of the $\gamma$ band along $\bar{\Gamma}-\bar{M}$ taken at different temperatures. Different colors represent different temperatures.

ARPES measurement. With an empirical value of the inner potential of approximately $8.2 \mathrm{eV}$ and $c=9.07 \AA$ [24], we find that $h v=86 \mathrm{eV}$ is close to the $\Gamma$ point and $100 \mathrm{eV}$ close to the $A$ point according to the free-electron final-state model [46]. Three electronlike pockets $(\alpha, \beta$, and $\kappa)$ along the $\bar{\Gamma}-\bar{K}$ direction are indicated in Figs. $1(\mathrm{~g})$ and $1(\mathrm{~h})$. All three bands show weak $k_{z}$ dispersions both at $E_{F}$ and $E_{F}-0.28 \mathrm{eV}$, as shown in Figs. 1(e) and 1(f), which reveal the 2D electronic dispersions along $\bar{\Gamma}-\bar{K}$ and 2D Dirac cones at the $\bar{K}$ points. Based on the ARPES data, we estimate that the widths of the $\alpha, \beta$, and $\kappa$ Fermi pockets along $\bar{\Gamma}-\bar{K}$ are about $0.42,0.10$, and $0.31 \AA^{-1}$, and their Fermi velocities are about $3.25,3.60$, and $1.70 \mathrm{eV} \AA$ (1.28 $\mathrm{eV} \AA$ for another branch of the Dirac bands), respectively.

Figure 2 shows the temperature evolution of the bands along the $\bar{\Gamma}-\bar{M}$ direction on a sample cleaved at $120 \mathrm{~K}$ (\#S1). The intensity plots along the $\bar{\Gamma}-\bar{M}$ direction taken at 120 and $10 \mathrm{~K}$ are shown in Figs. 2(a) and 2(b), respectively. Comparing the data taken at the two temperatures, one can see that the $\alpha$ band is shifted up, which is mainly attributed by surface reconstructions along with time [47]. Figure 2(c) shows the moment distribution curves (MDCs) of the $\alpha$ band taken at $120 \mathrm{~K}$, revealing the two splitting subbranches. The STM results suggest an isotropic scattering vector connecting different states of the $\alpha$ pocket [26-28], while the two $\mathrm{Sb}$ sites or $k_{z}$ integration can also cause the bands splitting in the ARPES data. We carry out substantial experiments on the samples with various conditions, e.g., cleaved at both high and low temperatures and then measure them at a few stabilized temperatures along with decreasing and increasing temperatures, as shown in the Supplemental Material Fig. S(1) [48]. Our data reveal that the $\alpha$ band at $\Gamma$ is sensitive to the sample surface and the vacuum, while the other bands are not.

At the $\bar{M}$ point, the $\kappa^{\prime}$ and $\gamma$ bands can be observed at $120 \mathrm{~K}$ [Fig. 2(d)]. The two bands are shifted down at $10 \mathrm{~K}$ and the $\delta$ band is brought out [Fig. 2(e)]. The $\delta$ band could be an edge state cut by the Fermi distribution function. We estimate that the widths of the $\alpha, \kappa^{\prime}$, and $\gamma$ Fermi pockets along $\bar{\Gamma}-\bar{M}$ at $10 \mathrm{~K}$ are about $0.42,0.22$, and $0.10 \AA^{-1}$ and their Fermi velocities are about 3.32, 1.68, and $4.20 \mathrm{eV} \AA$, respectively. We display in detail the temperaturedependent data in Figs. 2(f)-2(h). As shown in Fig. 2(f), one can find that the $\kappa^{\prime}$ and $\gamma$ bands begin to be shifted down around $T_{\mathrm{CDW}}(100 \mathrm{~K})$ and stand steadily around 


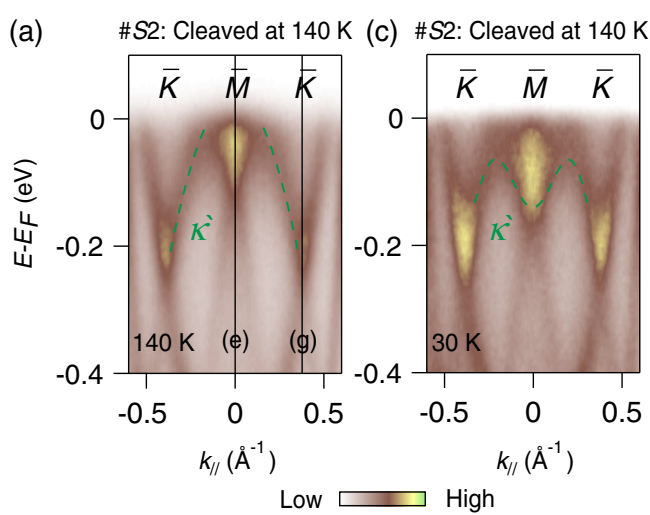

(b)

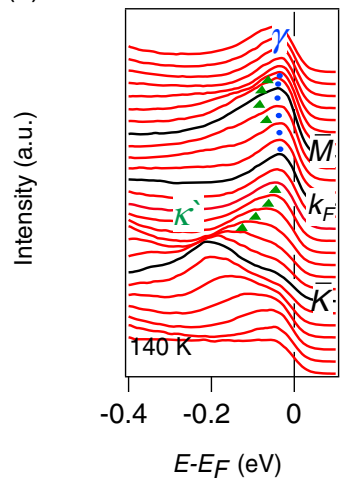

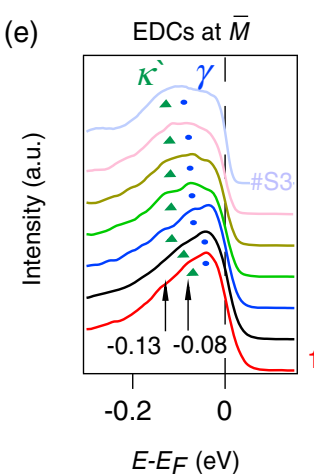

(d)

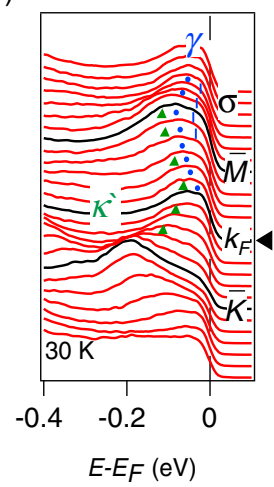

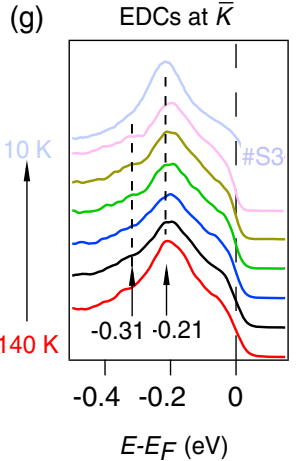

(f)

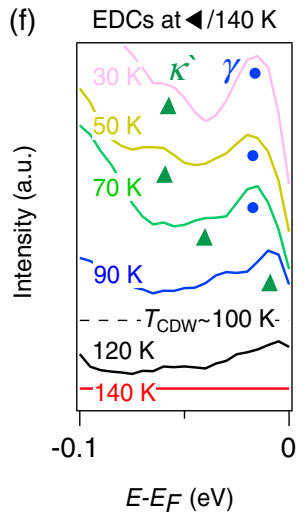

(h)
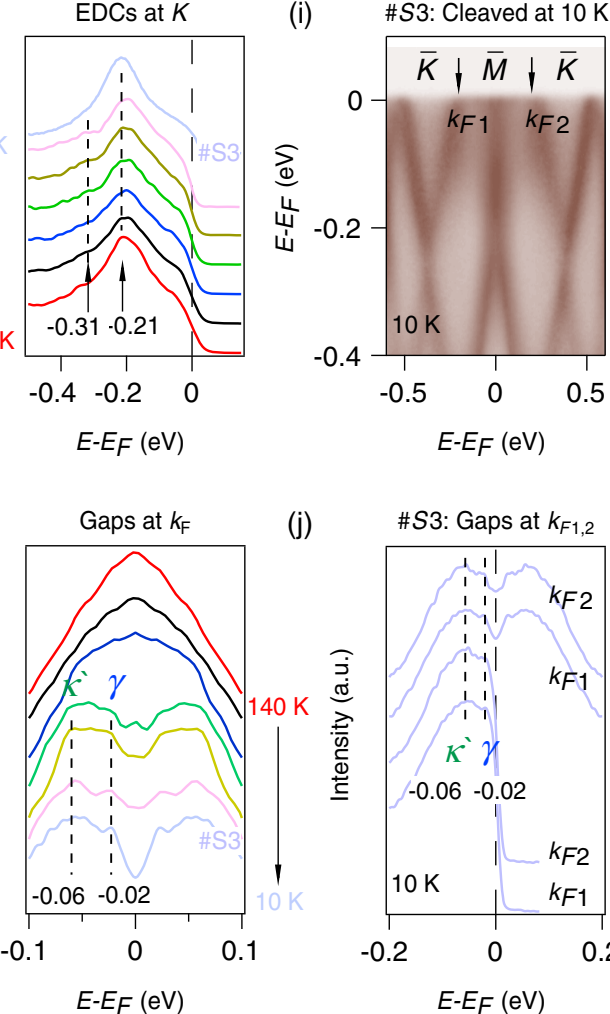

(j)

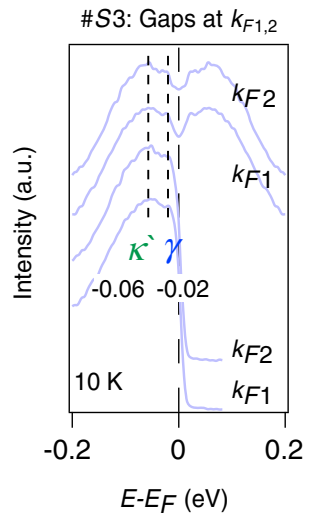

FIG. 3. (a)-(d) Intensity plots and corresponding EDCs along the $\bar{K}-\bar{M}$ direction taken at 140 and $30 \mathrm{~K}$, respectively. The sample (\#S2) is cleaved at $140 \mathrm{~K}$ and measured along with the decreasing temperature. The $\kappa^{\prime}$ and $\gamma$ bands are indicated by different color makers. (e) EDCs at the $\bar{M}$ center as indicated by the line in (a), taken at different temperatures. The energy positions are indicated by the black arrows. (f) EDCs at the fixed momentum [indicated by $\triangleleft$ in (d)] taken at different temperatures and divided by the EDC taken at $140 \mathrm{~K}$. (g) EDCs at the $\bar{K}$ center as indicated by the line in (a), taken at different temperatures. The energy positions are indicated by the black arrows. (h) The symmetrized EDCs at the $k_{F}$ of the $\gamma$ band along $\bar{K}-\bar{M}$ taken at different temperatures. The values of the gaps are marked. $\# S 3$ represents the EDCs taken on the third sample. (i) Intensity plot along the $\bar{K}-\bar{M}$ direction taken on the freshly cleaved sample at $10 \mathrm{~K}$ (\#S3). $k_{F}$ is indicated by the black arrows. (j) EDCs and their symmetrizations at the $k_{F}$ of the $\gamma$ band along $\bar{K}-\bar{M}$, as indicated in (i).

60-80 K with their band bottoms at 0.08 and $0.13 \mathrm{eV}$ below $E_{F}$, respectively. Below approximately $80 \mathrm{~K}$, the $\gamma$ band further opens the energy gap of about $20 \mathrm{meV}$, as shown in the energy distribution curves (EDCs) and their symmetrizations at $k_{F}$ [Figs. $2(\mathrm{~g})$ and $\left.2(\mathrm{~h})\right]$. While we do not observe the CDW gap opening at the $\alpha$ band centered at $\bar{\Gamma}$ at low temperatures along both $\bar{\Gamma}-\bar{M}$ and $\bar{\Gamma}-\bar{K}$, as shown in Supplemental Material Fig. S(2) [48].

Figures 3(a)-3(h) show the temperature evolution of the bands along the $\bar{K}-\bar{M}$ direction on a sample cleaved at $140 \mathrm{~K}$ (\#S2). From the intensity plots and the corresponding EDCs, one can clearly see that the bands near the $\bar{M}$ point are remarkably renormalized by CDW. The $\kappa^{\prime}$ band crosses $E_{F}$ at $140 \mathrm{~K}$ as shown in Figs. 3(a) and 3(b). The $\kappa^{\prime}$ and $\gamma$ bands shifting down along with the decreasing temperature mentioned above can be more clearly identified along the $\bar{K}-\bar{M}$ direction, as shown in Figs. 3(a)-3(e). More strikingly, the $\kappa^{\prime}$ band centered at $\bar{M}$ is flattened and sinks below $E_{F}$ at approximately $50-70 \mathrm{~K}$, as shown in Figs. 3(d) and 3(f), forming an M-shaped band with the tips of the $\kappa^{\prime}$ band (singularities) at about $60 \mathrm{meV}$ below $E_{F}$. The $\gamma$ band along $\bar{K}-\bar{M}$ also further opened the energy gap of about $20 \mathrm{meV}$, as shown in the symmetrized EDCs at the $k_{F}$ of Fig. 3(h). The temperature evolution of the $\kappa^{\prime}$ band seems like the calculation with an inverse Star of David pattern in Fig. 4(b).

To check the CDW-induced bands renormalization, we directly compare the data taken on the freshly cleaved samples at low temperature (\#S6 cleaved at $10 \mathrm{~K}$ ) and high temperature (\#S7 cleaved at $140 \mathrm{~K}$ ), as shown in the Supplemental Material Fig. S(3) [48]. The sharp contrast between Figs. $S(3)(a)$ and $S(3)(b)$ reveals that the band renormalization is indeed induced by the temperature rather than a trivial surface reconstruction. We also measure the energy gaps on a freshly cleaved sample at $10 \mathrm{~K}$ (\#S3), as shown in Figs. 3(i) and 3(j). The energy positions in Fig. 3(j) show CDW gaps at $-20 \mathrm{meV}$ and the tip of the $\kappa^{\prime}$ band at about $-60 \mathrm{meV}$, respectively. In addition, the temperature evolution of the Dirac cone at the $\bar{K}$ point is shown in Fig. 3(g). The Dirac point is almost not moved 

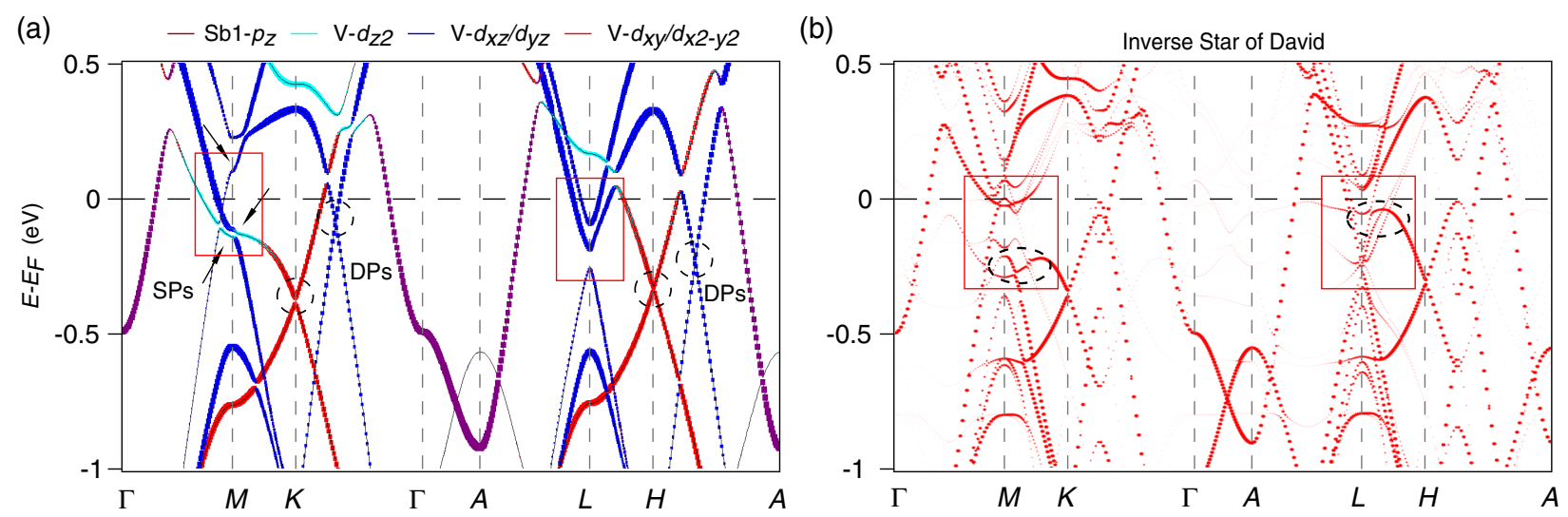

FIG. 4. (a) Orbital-projection band-structure calculation of $\mathrm{RbV}_{3} \mathrm{Sb}_{5}$ with spin-orbit coupling for the normal state. Here the main contribution orbitals near $E_{F}$ are shown, and the orbitals' weights are represented by both the colors and the size of the bands. The orbitals' weights of V atoms are the average values of the adjacent three V atoms. The saddle points (SPs) are indicated by the arrows, and the Dirac points (DPs) are marked with the dashed circles. The features at $\bar{M}(M$ and $L)$ are indicated by the red squares. (b) The unfolded band structure of the inverse Star of David phase. The bands with a strong renormalization at the $M$ and $L$ points are marked with the dashed ellipses. The marked band at $L$ sinks below $E_{F}$ in the CDW state.

along with decreasing temperature. The energy gap of the Dirac cone is about $100 \mathrm{meV}$, which is much larger than the calculated value of approximately $15-25 \mathrm{meV}$ induced by spin-orbit interaction in the normal state [Fig. 4(a)]. The CDW gaps induced by the band folding around the Dirac points along the $\bar{\Gamma}-\bar{K}$ direction [Fig. 4(b)] are needed to be considered.

With the help of the orbital-projection band calculation [Fig. 4(a) and the Supplemental Material Figs. S(4) [48]], one can find the $\alpha$ band at $\bar{\Gamma}$ is mainly contributed by the out-of-plane Sb1- $p_{z}$ (dark), and the $\kappa^{\prime}$ and $\gamma$ bands at $\bar{M}$ are mainly derived from the out-of-plane $\mathrm{V}-d_{z 2}$ (green) and $\mathrm{V}-d_{x z} / d_{y z}$ (blue) orbitals. The bonding of the out-of-plane orbitals and the interlayer coupling strength are enhanced along with decreasing the temperature, which is also revealed by the reduction of the $c$-axis lattice constant [21]. The renormalization of the bands with the out-ofplane character should be more appreciated with the CDW instability. Coulomb scattering of electrons between the orbital-selective saddle-point singularities at $M$ can give rise to instabilities of the Fermi surfaces and lead to CDW states [25-39]. The 2D Dirac bands at $\bar{K}\left(\kappa\right.$ and $\left.\kappa^{\prime}\right)$ originate from in-plane $\mathrm{V}-d_{x y} / d_{x 2-y 2}$ (red) orbitals, and hybridized with out-of-plane $\mathrm{V}-d_{x z} / d_{y z}$ (blue) orbitals near the $\bar{M}$ point. The Dirac bands remain nearly motionless upon the CDW phase transition.

The calculation in the CDW state with the inverse Star of David can well describe our experimental observations [Fig. 4(b) and the Supplemental Material Fig. S(5) [48]]. As marked with the dashed ellipses in Fig. 4(b), the $\kappa^{\prime}$ band at the $L$ point sinks below $E_{F}$, forming an M-shaped band with its tips at about $60 \mathrm{meV}$. The tips can be viewed as new singularities, which may be further associated with superconducting states. The CDW-induced bands renormalization is endowed with an electronic correlation effect. Previous studies provide strong evidence that traversing the singularity to $E_{F}$ is beneficial in the formation of ordering phenomena. For instance, in CDWmaterial $\mathrm{TaSe}_{2}$ also with 3D $2 \times 2 \times 2$ superstructure, by tuning the energy position of the singularity, the $T_{C}$ is enhanced by more than an order of magnitude [49]. Recently, thermal conductivity and high-pressure resistance measurements reveal two superconducting domes and exotic pairing states $[29,30,43,44]$, which may be associated with the optimal positions of the singularities concerning $E_{F}$ match with corresponding bosons.

In addition to the bands renormalization mentioned above, the CDW-induced energy gap $\Delta \sim 20 \mathrm{meV}$ is opened at $k_{F}$ of the band near $\bar{M}$ which is consistent with STM results [25-28]. The CDW-induced gap is not observed at the band near $\bar{\Gamma}$ at $T=10 \mathrm{~K}$ within the experimental energy resolution. Thus, momentum and orbital dependence of the electronic states are involved in the CDW formation in $\mathrm{RbV}_{3} \mathrm{Sb}_{5}$. The STM measurements further reveal that the CDW gap is particle-hole asymmetric [25-28], which is previously found in $\mathrm{CDW}$-material $\mathrm{NbSe}_{2}[50,51]$. As a case of typical quasi-2D materials without a strongly nested Fermi surface, the presence of a particle-hole asymmetric gap in $\mathrm{NbSe}_{2}$ could be an indication that electron correlation is important in driving the CDW [50,51]. Analogously, combined with the large ratio $2 \Delta / k_{B} T_{\mathrm{CDW}} \sim 4.55$ in analogy to strong-coupling superconductors, the CDW formation in $\mathrm{RbV}_{3} \mathrm{Sb}_{5}$ is likely mediated by electronic interactions enhanced by low dimensionality. Recent inelastic x-rayscattering studies demonstrate an unconventional and electronic driven mechanism that couples the $\mathrm{CDW}$ and the topological band structure in $\mathrm{RbV}_{3} \mathrm{Sb}_{5}$ [33].

In addition, as a $Z_{2}$ topological kagome metal, $\mathrm{RbV}_{3} \mathrm{Sb}_{5}$ hosts nontrivial topological Dirac surface states at the time-reversal-invariant $\bar{M}$ points and remains the same 
after the CDW transition, as shown in the Supplemental Material Fig. S(6) [48]. It is possible to realize the Majorana zero-energy modes and their related topological superconductivity in these materials. Because the electronlike bands near the $\bar{M}$ point show $k_{z}$ dispersions [see the Supplemental Material Fig. S(7) [48]], the surface states at $\bar{M}$ are possibly located above $E_{F}$. The chemical potential needs to be elevated for further study of the surface states in detail.

\section{CONCLUSION}

In summary, we study the electronic structures of a kagome superconductor $\mathrm{RbV}_{3} \mathrm{Sb}_{5}$ in both the normal phase and the CDW phase. We observe the CDW-induced bands renormalization and energy gaps on the bands at the zone boundary, where multiple orbital-selective singularities exist. Momentum and orbital dependence of the electronic states are involved in the CDW formation and the associated superconductivity. Our findings strongly imply that the singularities near $E_{F}$ play important roles in the formation of ordering phases and the electronic states on the residual Fermi surfaces to the superconducting pairing.

\section{ACKNOWLEDGMENTS}

This work is supported by the National Key R\&D Program of the Ministry of Science and Technology of China (MOST) (Grants No. 2018YFE0202600, No. 2017YFA0302903, and No. 2016YFA0300504), the National Natural Science Foundation of China (NSFC) (Grants No. 11822412, No. 11774421, No. 11774423, and No. 11774424), the Beijing Natural Science Foundation (Grant No. Z200005), the CAS Interdisciplinary Innovation Team, the Fundamental Research Funds for the Central Universities, the Research Funds of Renmin University of China (RUC) (Grants No. 18XNLG14, No. 19XNLG03, and No. 19XNLG17), and the Beijing National Laboratory for Condensed Matter Physics. N. Z. is supported by the Outstanding Innovative Talents Cultivation Funded Programs 2021 of the RUC. The ARPES experiments are performed on the Dreamline beam line of the Shanghai Synchrotron Radiation Facility (SSRF) and supported by MOST (Grant No. 2016YFA0401002), and the 03U beam line of the SSRF is supported by the ME2 project (Grant No. 11227902) from the NSFC. Computational resources are provided by the Physical Laboratory of HighPerformance Computing at the RUC and Shanghai Supercomputer Center.

Z.L., K. L., H.L., and S.W. provided strategy and advice for the research. Z. L., M.L., W.S., Z. L., D. S., Y.H., and S.W. performed ARPES measurements; N.Z. and K. L. carried out the calculations; Q. Y., C. G., Z. T., and H.L. synthesized the single crystals. All authors contributed to writing the manuscript. Z.L., N.Z., and Q. Y. contributed equally to this work.

\section{APPENDIX: METHODS}

Single crystals of $\mathrm{RbV}_{3} \mathrm{Sb}_{5}$ are synthesized by the selfflux method as described elsewhere [23]. $\mathrm{RbV}_{3} \mathrm{Sb}_{5}$ single crystals are stable in the air. ARPES measurements are performed at the Dreamline and 03U beam lines of the SSRF. The energy and angular resolutions are set to 10-24 meV and $0.02 \AA^{-1}$, respectively. The Fermi cutoff of the samples is referenced to an evaporated gold film on the sample holder. Samples are cleaved in situ, exposing flat mirrorlike (001) surfaces. The pressure is maintained at less than $2 \times 10^{-10}$ Torr during temperature-dependent measurements.

The first-principles electronic structure calculations on $\mathrm{RbV}_{3} \mathrm{Sb}_{5}$ are performed by using the projector-augmentedwave method [52,53] as implemented in the Vienna $a b$ initio simulation package [54]. The generalized-gradient approximation of Perdew-Burke-Ernzerhof type [55] is used for the exchange-correlation functional. The kinetic energy cutoff of the plane-wave basis is set to $350 \mathrm{eV}$. The $\mathrm{BZ}$ is sampled with a $10 \times 10 \times 6 k$-point mesh. For the Fermi-surface broadening, the Gaussian smearing method with a width of $0.05 \mathrm{eV}$ is adopted. The zero-damping DFTD3 method is adopted to describe the interlayer van der Waals interactions [56]. The lattice constants and the atomic positions are fully relaxed until the forces on all atoms are smaller than $0.01 \mathrm{eV} / \AA$. The relaxed lattice constants $a=b=5.4333 \AA$ and $c=8.9986 \AA$ are consistent with the experimental result [24]. The surface states in the projected $2 \mathrm{D} \mathrm{BZ}$ are calculated with the surface Green's function method by using the WannierTools package [57]. The tight-binding Hamiltonian of the semi-infinite system is constructed by the maximally localized Wannier functions [58]. To study the CDW phase of $\mathrm{RbV}_{3} \mathrm{Sb}_{5}$, a $2 \times 2 \times 1$ supercell and a $5 \times 5 \times 5 k$-point mesh for the corresponding BZ sampling are used. The initial atomic distortions are first set according to the in-plane structures of the previously reported Star of David and inverse Star of David patterns [34], and then both the lattice parameters and the internal atomic positions are fully relaxed. The band structures of the CDW phases are unfolded in the BZ of the unit cell with the band-unfolding method [59] as in the PyVaspwfc package [60].

[1] Z. Liu et al., Orbital-Selective Dirac Fermions and Extremely Flat Bands in Frustrated Kagome-Lattice CoSn, Nat. Commun. 11, 4002 (2020).

[2] K. Kuroda et al., Evidence for Magnetic Weyl Fermions in a Correlated Metal, Nat. Mater. 16, 1090 (2017).

[3] L. Ye et al., Massive Dirac Fermions in a Ferromagnetic Kagome Metal, Nature (London) 555, 638 (2018).

[4] K. Kim et al., Large Anomalous Hall Current Induced by Topological Nodal Lines in a Ferromagnetic van der Waals Semimetal, Nat. Mater. 17, 794 (2018). 
[5] E. Liu et al., Giant Anomalous Hall Effect in a Ferromagnetic Kagome-Lattice Semimetal, Nat. Phys. 14, 1125 (2018).

[6] Q. Wang, Y. Xu, R. Lou, Z. Liu, M. Li, Y. Huang, D. Shen, H. Weng, S. Wang, and H. Lei, Large Intrinsic Anomalous Hall Effect in Half-Metallic Ferromagnet $\mathrm{Co}_{3} \mathrm{Sn}_{2} \mathrm{~S}_{2}$ with Magnetic Weyl Fermions, Nat. Commun. 9, 3681 (2018).

[7] I. Belopolski et al., Discovery of Topological Weyl Fermion Lines and Drumhead Surface States in a Room Temperature Magnet, Science 365, 1278 (2019).

[8] D. F. Liu et al., Magnetic Weyl Semimetal Phase in a Kagome Crystal, Science 365, 1282 (2019).

[9] N. Morali, R. Batabyal, P. K. Nag, E. Liu, Q. Xu, Y. Sun, B. Yan, C. Felser, N. Avraham, and H. Beidenkopf, Fermi-Arc Diversity on Surface Terminations of the Magnetic Weyl Semimetal $\mathrm{Co}_{3} \mathrm{Sn}_{2} \mathrm{~S}_{2}$, Science 365, 1286 (2019).

[10] M. Kang et al., Dirac Fermions and Flat Bands in the Ideal Kagome Metal FeSn, Nat. Mater. 19, 163 (2020).

[11] Z. Lin, C. Wang, P. Wang, S. Yi, L. Li, Q. Zhang, Y. Wang, Z. Wang, H. Huang, Y. Sun et al., Dirac Fermions in Antiferromagnetic FeSn Kagome Lattices with Combined Space Inversion and Time-Reversal Symmetry, Phys. Rev. B 102, 155103 (2020).

[12] M. Li et al., Dirac Cone, Flat Band and Saddle Point in Kagome Magnet $\mathrm{YMn}_{6} \mathrm{Sn}_{6}$, Nat. Commun. 12, 3129 (2021).

[13] Z. Lin, J. H. Choi, Q. Zhang, W. Qin, S. Yi, P. Wang, L. Li, Y. Wang, H. Zhang, Z. Sun et al., Flatbands and Emergent Ferromagnetic Ordering in $\mathrm{Fe}_{3} \mathrm{Sn}_{2}$ Kagome Lattices, Phys. Rev. Lett. 121, 096401 (2018).

[14] J.-X.Yin et al., Giant and Anisotropic Many-Body Spin Orbit Tunability in a Strongly Correlated Kagome Magnet, Nature (London) 562, 91 (2018).

[15] Y. Zhang et al., Emergence of Kondo Lattice Behavior in a van der Waals Itinerant Ferromagnet, $\mathrm{Fe}_{3} \mathrm{GeTe}_{2}$, Sci. Adv. 4, eaao6791 (2018).

[16] J.-X. Yin et al., Negative Flat Band Magnetism in a SpinOrbit-Coupled Correlated Kagome Magnet, Nat. Phys. 15, 443 (2019).

[17] J.-X. Yin et al., Quantum-Limit Chern Topological Magnetism in $\mathrm{TbMn}_{6} \mathrm{Sn}_{6}$, Nature (London) 583, 533 (2020).

[18] T. M. Rice and G. K. Scott, New Mechanism for a ChargeDensity-Wave Instability, Phys. Rev. Lett. 35, 120 (1975).

[19] S. Manzeli, D. Ovchinnikov, D. Pasquier, O. V. Yazyev, and A. Kis, 2D Transition Metal Dichalcogenides, Nat. Rev. Mater. 2, 17033 (2017).

[20] B. R. Ortiz et al., New Kagome Prototype Materials: Discovery of $\mathrm{KV}_{3} \mathrm{Sb}_{5}, \mathrm{RbV}_{3} \mathrm{Sb}_{5}$, and $\mathrm{CsV}_{3} \mathrm{Sb}_{5}$, Phys. Rev. Mater. 3, 094407 (2019).

[21] B. R. Ortiz et al., $\mathrm{CsV}_{3} \mathrm{Sb}_{5}: A \mathbb{Z}_{2}$ Topological Kagome Metal with a Superconducting Ground State, Phys. Rev. Lett. 125, 247002 (2020).

[22] S.-Y. Yang et al., Giant, Unconventional Anomalous Hall Effect in the Metallic Frustrated Magnet Candidate, $\mathrm{KV}_{3} \mathrm{Sb}_{5}$, Sci. Adv. 6, eabb6003 (2020).

[23] Q. Yin, Z. Tu, C. Gong, Y. Fu, S. Yan, and H. Lei, Superconductivity and Normal-Stat Properties of Kagome Metal $\mathrm{RbV}_{3} \mathrm{Sb}_{5}$ Single Crystals, Chin. Phys. Lett. 38, 037403 (2021).
[24] B. R. Ortiz, P. M. Sarte, E. M. Kenney, M. J. Graf, S. M. L. Teicher, R. Seshadri, and S. D. Wilson, Superconductivity in the $\mathbb{Z}_{2}$ Kagome Metal $\mathrm{KV}_{3} \mathrm{Sb}_{5}$, Phys. Rev. Mater. 5, 034801 (2021).

[25] Y.-X. Jiang et al., Discovery of Topological Charge Order in Kagome Superconductor $\mathrm{KV}_{3} \mathrm{Sb}_{5}$, Nat. Mater., DOI: https://doi.org/10.1038/s41563-021-01034-y (2021).

[26] H. Zhao et al., Cascade of Correlated Electron States in a Kagome Superconductor $\mathrm{CsV}_{3} \mathrm{Sb}_{5}$, arXiv:2103.03118.

[27] Z. Liang et al., Three-Dimensional Charge Density Wave and Robust Zero-Bias Conductance Peak inside the Superconducting Vortex Core of a Kagome Superconductor $\mathrm{CsV}_{3} \mathrm{Sb}_{5}$, Phys. Rev. X 11, 031026 (2021).

[28] H. Chen et al., Roton Pair Density Wave and Unconventional Strong-Coupling Superconductivity in a Topological Kagome Metal, arXiv:2103.09188.

[29] C. C. Zhao et al., Nodal Superconductivity and Superconducting Domes in the Topological Kagome Metal $\mathrm{CsV}_{3} \mathrm{Sb}_{5}$, arXiv:2102.08356.

[30] F. Y. Chen et al., Double Superconducting Dome and Triple Enhancement of $T_{c}$ in the Kagome Superconductor $\mathrm{CsV}_{3} \mathrm{Sb}_{5}$ under High Pressure, Phys. Rev. Lett. 126, 247001 (2021).

[31] E. Uykur, B. R. Ortiz, S. D. Wilson, M. Dressel, and A. A. Tsirlin, Optical Detection of Charge-Density-Wave Instability in the Non-Magnetic Kagome Metal $\mathrm{KV}_{3} \mathrm{Sb}_{5}$, arXiv: 2103.07912.

[32] Y. Wang et al., Proximity-Induced Spin-Triplet Superconductivity and Edge Supercurrent in the Topological Kagome Metal $\mathrm{K}_{1-x} \mathrm{~V}_{3} \mathrm{Sb}_{5}$, arXiv:2012.05898.

[33] H.X. Li et al., Observation of Unconventional Charge Density Wave without Acoustic Phonon Anomaly in Kagome Superconductors $A \mathrm{~V}_{3} \mathrm{Sb}_{5}(A=\mathrm{Rb}, \mathrm{Cs})$, Phys. Rev. X 11, 031050 (2021).

[34] H. Tan, Y. Liu, Z. Wang, and B. Yan, Charge Density Waves and Electronic Properties of Superconducting Kagome Metals, Phys. Rev. Lett. 127, 046401 (2021).

[35] X. Feng, K. Jiang, Z. Wang, and J. Hu, Chiral Flux Phase in the Kagome Superconductor $\mathrm{KV}_{3} \mathrm{Sb}_{5}$, Sci. Bull. 66, 1384 (2021).

[36] S.-L. Yu and J.-X Li, Chiral Superconducting Phase and Chiral Spin-Density-Wave Phase in a Hubbard Model on the Kagome Lattice, Phys. Rev. B 85, 144402 (2012).

[37] W.-S. Wang, Z.-Z. Li, Y.-Y. Xiang, and Q.-H Wang, Competing Electronic Orders on Kagome Lattices at van Hove Filling, Phys. Rev. B 87, 115135 (2013).

[38] M. L. Kiesel and R. Thomale, Sublattice Interference in the Kagome Hubbard Model, Phys. Rev. B 86, 121105(R) (2012).

[39] M. L. Kiesel, C. Platt, and R. Thomale, Unconventional Fermi Surface Instabilities in the Kagome Hubbard Model, Phys. Rev. Lett. 110, 126405 (2013).

[40] M. Denner, R. Thomale, and T. Neupert, Analysis of Charge Order in the Kagome Metal $A_{V_{3}} \mathrm{Sb}_{5}(A=\mathrm{K}, R b, C s)$, arXiv:2103.14045.

[41] S. Ni et al., Anisotropoic Superconducting Properties of Kagome Metal $\mathrm{CsV}_{3} \mathrm{Sb}_{5}$, Chin. Phys. Lett. 38, 057403 (2021).

[42] W. Duan et al., Nodeless Superconductivity in the Kagome Metal $\mathrm{CsV}_{3} \mathrm{Sb}_{5}$, arXiv:2103.11796. 
[43] Z. Zhang et al., Pressure-Induced Reemergence of Superconductivity in Topological Kagome Metal $\mathrm{CsV}_{3} \mathrm{Sb}_{5}$, Phys. Rev. B 103, 224513 (2021).

[44] X. Chen, X. Zhan, X. Wang, J. Deng, X.-B. Liu, X. Chen, J.-G. Guo, and X. Chen, Highly-Robust Reentrant Superconductivity in $\mathrm{CsV}_{3} \mathrm{Sb}_{5}$ under Pressure, Chin. Phys. Lett. 38, 057402 (2021).

[45] E. M. Kenney et al., Absence of Local Moments in the Kagome Metal $\mathrm{KV}_{3} \mathrm{Sb}_{5}$ as Determined by Muon Spin Spectroscopy, arXiv:2012.04737.

[46] Z. H. Liu et al., Three Dimensionality and Orbital Characters of the Fermi Surface in (Tl, $\mathrm{Rb})_{y} \mathrm{Fe}_{2-x} \mathrm{Se}_{2}$, Phys. Rev. Lett. 109, 037003 (2012).

[47] Y. Luo et al., Distinct Band Reconstructions in Kagome Superconductor $\mathrm{CsV}_{3} \mathrm{Sb}_{5}$, arXiv:2106.01248.

[48] See Supplemental Material at http://link.aps.org/ supplemental/10.1103/PhysRevX.11.041010 for additional data of the ARPES experiments and the first-principles calculations.

[49] A. Chikina, A. Fedorov, D. Bhoi, V. Voroshnin, E. Haubold, Y. Kushnirenko, K. H. Kim, and S. Borisenko, Turning Charge-Density Waves into Cooper Pairs, npj Quantum Mater. 5, 22 (2020).

[50] A. Soumyanarayanan, M. M. Yee, Y. He, J. van Wezel, D. J. Rahn, K. Rossnagel, E. W. Hudson, M. R. Norman, and J.E. Hoffman, Quantum Phase Transition from Triangular to Stripe Charge Order in $\mathrm{NbSe}_{2}$, Proc. Natl. Acad. Sci. U.S.A. 110, 1623 (2013).

[51] U. Chatterjee et al., Emergence of Coherence in the ChargeDensity Wave State of $2 \mathrm{H}-\mathrm{NbSe}_{2}$, Nat. Commun. 6, 6313 (2015).

[52] P. E. Blöchl, Projector Augmented-Wave Method, Phys. Rev. B 50, 17953 (1994).
[53] G. Kresse and D. Joubert, From Ultrasoft Pseudopotentials to the Projector Augmented-Wave Method, Phys. Rev. B 59, 1758 (1999).

[54] G. Kresse and J. Furthmüller, Efficient Iterative Schemes for Ab Initio Total-Energy Calculations Using a Plane-Wave Basis Set, Phys. Rev. B 54, 11169 (1996).

[55] J. P. Perdew, K. Burke, and M. Ernzerhof, Generalized Gradient Approximation Made Simple, Phys. Rev. Lett. 77, 3865 (1996).

[56] S. Grimme, J. Antony, S. Ehrlich, and H. Krieg, A Consistent and Accurate Ab Initio Parametrization of Density Functional Dispersion Correction (DFT-D) for the 94 Elements $\mathrm{H}-\mathrm{Pu}$, J. Chem. Phys. 132, 154104 (2010).

[57] Q. Wu, S. Zhang, H.-F. Song, M. Troyer, and A. Soluyanov, WannierTools: An Open-Source Software Package for Novel Topological Materials, Comput. Phys. Commun. 224, 405 (2018).

[58] A. A. Mostofi, J. R. Yates, G. Pizzi, Y.-S. Lee, I. Souza, D. Vanderbilt, and N. Marzari, An Updated Version of Wannier90: A Tool for Obtaining Maximally-Localised Wannier Functions, Comput. Phys. Commun. 185, 2309 (2014).

[59] V. Popescu and A. Zunger, Extracting e versus $k$ Effective Band Structure from Supercell Calculations on Alloys and Impurities, Phys. Rev. B 85, 085201 (2012).

[60] Q. J.Zheng, https://github.com/QijingZheng/VaspBandUnfolding (2018).

Correction: The seventh and eighth sentences of the fifth paragraph of Sec. II contained errors and have been set right. 